\title{
The management of oral erythema multiforme in juvenile patient
}

\author{
Diah Savitri Ernawati \\ Department of Oral Medicine \\ Faculty of Dentistry, Airlangga University
}

Surabaya - Indonesia

\begin{abstract}
Erythema multiforme is an acute inflammatory disease of the skin and mucous membranes that causes a variety of the skin lesionhence the name 'multiforme'. The oral mucosa looks severely inflamed, but the feature are non specific and usually a biopsy is required in order to confirm the diagnosis. Cracked, bleeding, Crusted, swollen and ulcers of the lips is very characteristic of erythema multiforme, and lip involvement may cause significant morbidity. EM is assumed as an immune complex disorder which rises as a result of an immune response to an external agent such as herpes simplex virus or various drugs. We reported: 14-year girl, complained she suffered from painful oral ulceration for one week. One weeks advance the patient received a treatment of paracetamol and paramex for febris, headache and cough. Clinical examination of the skin showed no signs of cutaneous involvement. Other site such as the conjunctival, and genital were also free of lesions. The patients had several red-based superficial erosions on the upper and lower lips accompanied by crusting and bleeding. Intra oral findings showed multiple irregular erosions, ulcers and intense erythematous areas, mainly on the labial mucosa. The clinical diagnosis of EM was concluded by anamnesis and clinical appearance, with differential diagnosis of secondary herpes infection (herpes labialis) and pemphigus vulgaris. Systemic and topical corticosteroid therapy is frequently used to treat EM Although it may partially suppress the disease. Objective: This report explains and describes the management of patients with EM which may help dentists to determine an accurate diagnosis to avoid further complication and to give medical intervention to the disease. Conclusion: Early recognition of this disease may prevent delayed diagnosis and incorrect treatment.
\end{abstract}

Key words: oral erythema multiforme, children, oral management

Correspondence: Diah Savitri Ernawati, c/o: Bagian Oral Medicine, Fakultas Kedokteran Gigi Universitas Airlangga. Jln. Mayjend. Prof. Dr. Moestopo no. 47 Surabaya 60132, Indonesia. E-mail: savitri_glx@yahoo.com

\section{INTRODUCTION}

Erythema multiforme (EM) is an acute inflammatory disease of the skin and mucous membranes. The main feature of an attack is the sudden development of widespread erosions of the oral mucosa which characteristically involving the lips. The erosions are produced by the disintegration of sub epidermal bullae, lesions that only rarely last long enough to become a diagnostic feature. ${ }^{1}$

Erythema multifome is an immune-mediated disease that may be initiated either by deposition of immune complexes in the superficial micro vessels of skin and mucosa, or cell-mediated immunity. The most common triggers for episodes of EM are herpes simplex virus and drug eruption. The drugs that most frequently associated with EM reactions are oxycam non steroidal anti inflamatory drugs (NSAIDs), sulfonamides; anticonvulsants such as carbamazepine; Phenobarbital; and phenytoin; trimethoprim-sulfonmide combinations, allopurinol and penicillin. ${ }^{1,2}$ This report explains and describe a case of oral erythema multiforme in juvenile patients and its management.

Erythema Multiforme is most frequently detected in children and young adults and is rarely found after the age of 50 years. It has an acute or even an explosive onset and generalized symptoms such as fever and malaise, which appear in severe cases. A patient may also be asymptomatic and in less than 24 hours have extensive lesions of the skin and mucosa. Erythema multiforme is a self-limiting form of the disease and is characterized by macules and papules $0.5-2 \mathrm{~cm}$ in diameter, appearing in a symmetric distribution. The most common skin areas involved are the hands, feet and extensor surfaces of the elbows and knees. The face and the neck are commonly involved. ${ }^{1,2,3}$ Typical skin lesions of EM may be non specific macules, papules and vesicles. More typical skin lesions contain petechiae in the center of the lesions. The pathognomonic lesion is the target or "iris" lesions, which consists of central bulla or pale clearing area surrounded by edema and erythematous bands. EM is classified as Stevens-Johnson's syndrome when the generalized vesicles and bullae involve the skin, mouth, eyes and genitals. ${ }^{1,2,3}$

The diagnosis is made on the basis of the total clinical picture, including the rapid onset of lesion. The oral lesion starts as bullae on an erythematous base, but intact bullae are rarely seen by the clinician because they break rapidly into irregular ulcers. EM lesion is larger and deeper than common ulcers and often bleeds spontaneously or at the slightest touch. Lesions may occur any where on the oral mucosa, but involvement of the lips is especially prominent, and gingival involvement is rare. This is an 
important criterion for distinguishing EM from primary herpes simplex infection. In full blown clinical cases, the lips are extensively eroded, and large portions of the oral mucosa are denuded of epithelium. The patient can hardly eat or even swallow and tend to drool blood-tinged saliva. Within 2 or 3 days, the labial lesions begin to crust. Healing occurs within two weeks in majority of cases, but in some severe cases extensive disease may continue for several weeks. ${ }^{2,3}$

Histopathological examination of ulcer reveals intense chronic inflammatory infiltrate. Degenerative changes in the epithelium are associated with infiltration by inflammatory cells which also involve the corium and may have a perivascular distribution. Leakage of immunoglobulins from blood vessels has been reported, but vasculitis is not seen histologically. ${ }^{1}$

Treatment of the case is restricted to the mouth area depending on the use of local or systemic steroids, to which there is usually a rapid response. A steroid mouthwash is likely to give symptomatic relief and effectively reverse the process in a few days. When the skin or oral lesions are severely damaged or when the eye or other mucous membranes are affected, a short course of systemic steroids may be necessary to shorten the attack. An initial dose of 30 $\mathrm{mg} /$ day to $50 \mathrm{mg}$ /day of prednisone or methyl prednisolone for several days, which is then tapered, is helpful to quicken the healing time of EM, particularly when therapy started early in the course of the disease., ${ }^{2,3}$

\section{CASES}

Case 1: A 14-year girl was referred to the Oral Medicine Clinic, School of Dentistry of Airlangga University, with a one week history of painful oral ulceration. A week in advance the patient received a treatment of paracetamol and paramex for febris and headache and cough. The oral lesions appeared a few days later, causing considerable discomfort and affected her normal oral function. Personal and family histories and laboratory tests were uneventful.

Clinical examination of the skin showed no signs of cutaneous involvement. Other site such as the conjunctival, and genital were also free of lesions. The patients had several red-based superficial erosions on the upper and lower lips accompanied by crusting and bleeding. Intra oral findings showed multiple irregular erosions, ulcers and intense erythematous areas, mainly on the labial mucosa (Figure 1). The clinical diagnosis of EM was concluded by anamnesis and clinical appearance, with differential diagnosis of secondary herpes infection (herpes labialis) and pemphigus vulgaris.

The patient was treated with local and systemic prednisone (30 mg/day). The application of topical steroids was necessary due to the severity of the lesions in the upper and lower lip. The disease showed a marked improvement in two weeks.
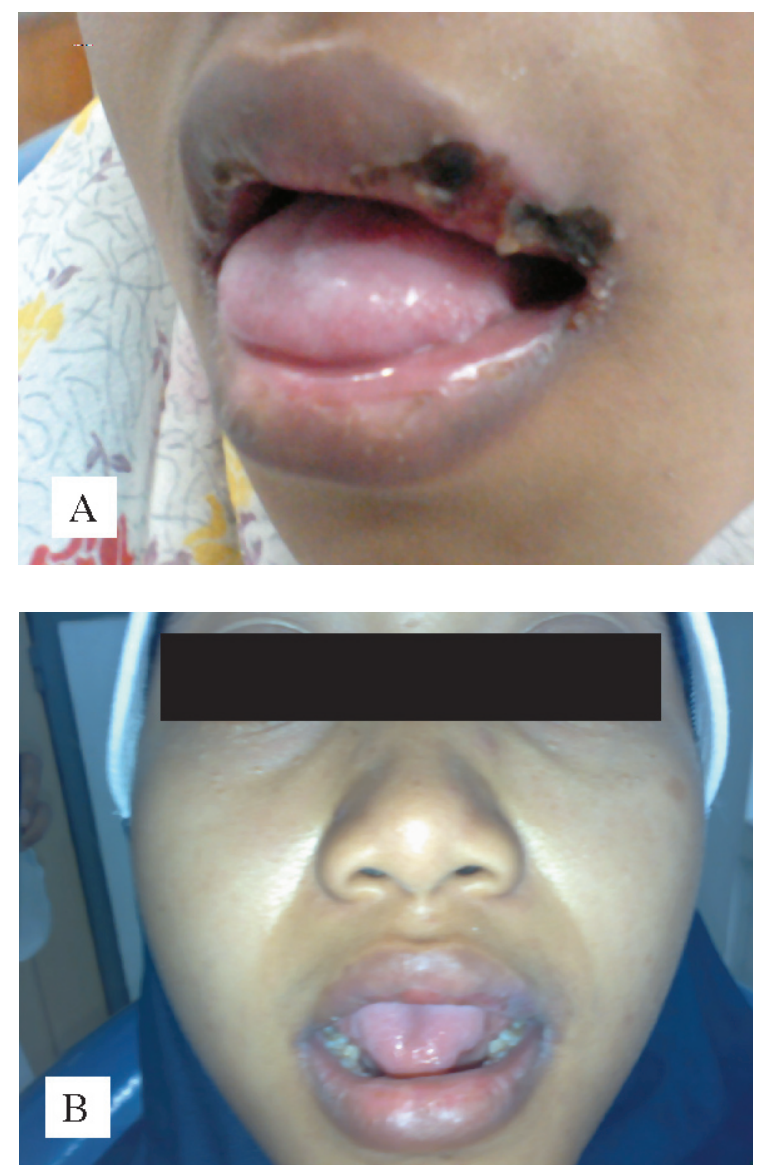

Figure 1. (A) Erythema multiforme. On the first visit: Ulceration of the vermillion border of the lip with bleeding, swelling and crusting is characteristic. (B) Twelve days after the fist visit, patients came with much better condition.

Case 2: A 7-year old boy was referred to the Oral Medicine Clinic, Faculty of Dentistry Airlangga University, after two week history of painful oral ulceration. One week in advance, the patient received a treatment of $250 \mathrm{mg}$ of amoxicillin and $250 \mathrm{mg}$ of paracetamol for laryngitis. The oral lesions appeared a few days later, causing considerable discomfort and affected his normal oral function. He also reported weight loss during this time since the ulcers caused eating difficulty.

Clinical examination, Intra oral findings showed multiple irregular erosions, ulcers and intense erythematous areas, mainly on the labial mucosa. The skin showed no signs of cutaneous involvement. Others site such as the conjunctival, and genital were also free of lesions. The patients had several red-based superficial erosions on the upper and lower lips accompanied by crusting and bleeding (Figure 2). The patient was commenced on systemic corticosteroids (prednisolone) at an initial dose $30 \mathrm{mg} /$ day. The application of topical steroids was necessary due to the severity of the lesions in the upper and lower lip. The disease showed a marked improvement in two weeks. 

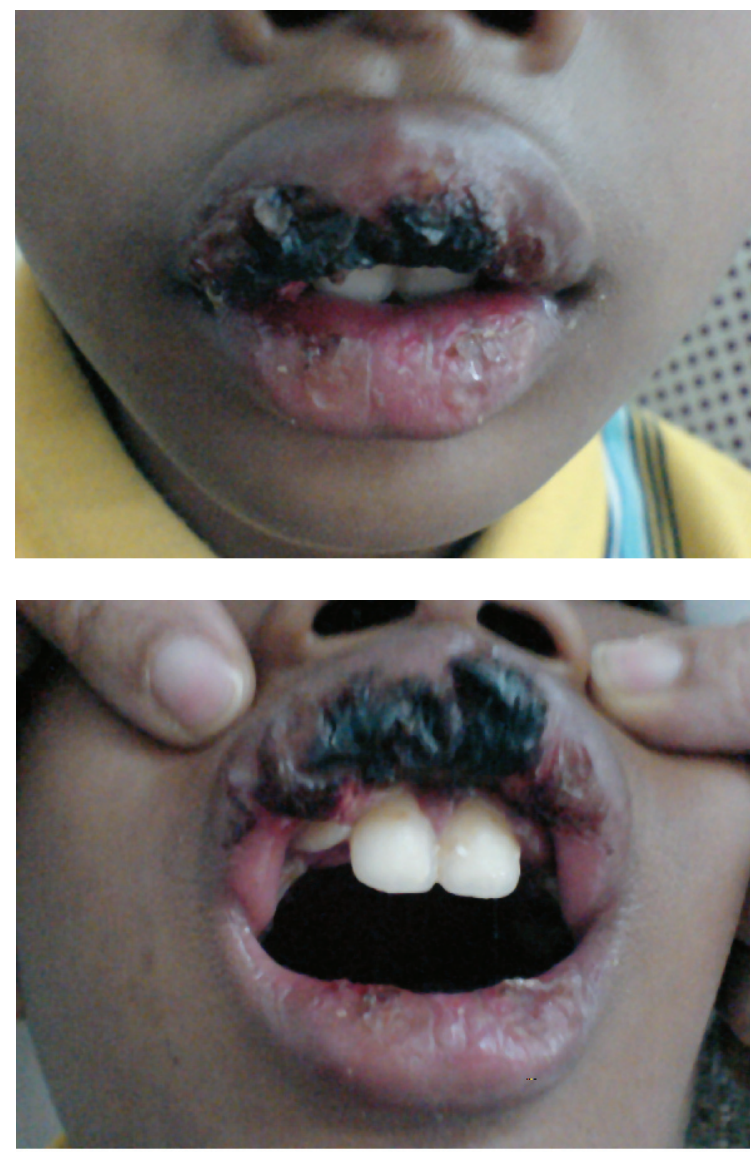

Figure 2. Erythema multiforme. Ulceration of the vermillion border of the lip with bleeding, swelling and crusting is characteristic.

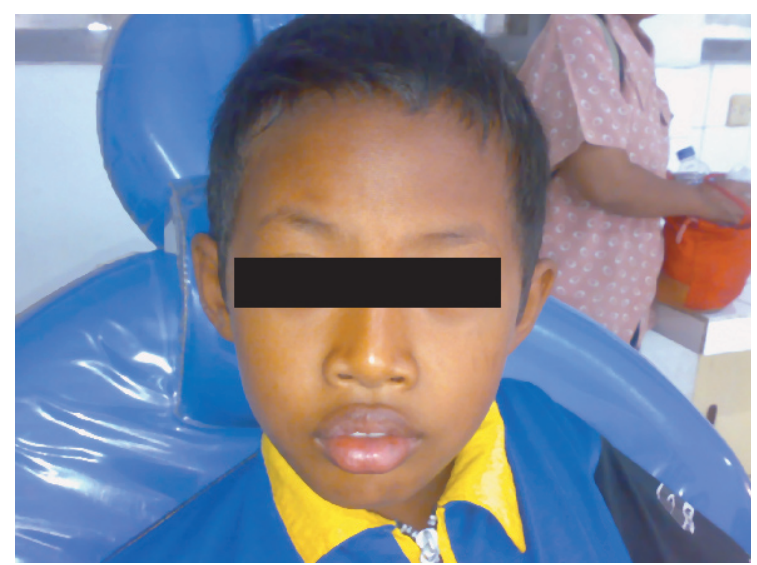

Figure 3. On the $3^{\text {rd }}$ visit, a week later, the lesions had completely healed and the lips appear normal.

\section{CASE MANAGEMENT}

On the first visit, the lips were sanitized with sterile gauze and antiseptic solution. Patient was prescribed a topical cream consisting 0.1 gram of Hidrocortisone,
0.25 gram of Lanoline, 0.1 gram of Kemicitine, and 10 gram of Vaseline, which should be applied on the oral lesions. Other medication are systemic intermediateacting corticosteroid $30 \mathrm{mg}$ per day for 5 days and multivitamin. ${ }^{2,4}$

Six days later the patient showed up with less complaint. The prescribed medicine was consumed as instructed. Pain on the lips and mouth lessened. Clinical examination revealed improved condition. Crust on upper lips have mostly eroded, the swelling lessened, and ulcer in the labial mucosa has significantly healed. The systemic prednisone dose was then tapered while the topical corticosteroid was maintained.

Six days after the $1^{\text {st }}$ control, or twelve days after the $1^{\text {st }}$ visit, patient came with much better condition. Crusts on the upper lip had completely eroded, leaving the lips in slight erythematous condition, sensitive but not painful. The lower lip appeared normal. On the $3^{\text {rd }}$ visit, a week later, the lesions had completely healed and the lips appear normal (Figure 3).

\section{DISCUSSION}

Erythema multiforme is an acute, self-limiting, mucocutaneous disorder with symmetrically distributed, erythematous skin lesions, some with concentric colour changes (target lesions), which resolve within 1 to 6 weeks and show compatible histology. There exists a subgroup of patients with recurrent EM in whom frequent episodes of the disease over several years cause significant morbidity. The experience 2 or more attacks per year.

Prodromal symptoms include malaise, fever, headache, sore throat, rhinorrhoea and cough which may occur approximately 1 week before the onset of EM. The typical primary lesions of EM is a round, erythematous macule that rapidly becomes popular or urticarial. Individual oedematous papules may enlarge to small plaques and may also develop concentric alteration in morphology and colour. The concentric changes produce characteristic lesions with either a central blister or a central area of necrosis resulting in target lesions. As the skin lesions resolve, they may develop some scaling but typically heal without theraphy. 3

The definite etiology remains unclear. No convincing mechanism has been proposed, nevertheless the disease may be a reaction to various causes. Infection, particularly herpetic, may serve as triggering factors. Drugs, particularly sulphonamides and barbiturate, have also been reported as a trigger. A positive drug history is also rare. Even when drugs have been taken, coincidence cannot always be excluded and in most patients no precipitating cause can be found. ${ }^{3,6}$

The main feature of an attack is the sudden development of widespread erosions of the oral mucosa, characteristically involving the lips. The erosions are produced by the disintegration of sub epidermal bullae, lesions that only 
rarely last long enough to become a diagnostic feature. The erosions on the lips (especially the lower lip) are accompanied by crusting and bleeding and are, if not absolutely diagnostic, strong pointers to the nature of the condition. There is often a cervical lymphadenitis with pyrexia and the patient feels unwell.

The lesions are typically symmetrical and occur commonly on the dorsal surfaces of the hands and extensor aspects of the extremities. Mucosal involvement occurs in $25 \%$ to $60 \%$ of cases either simultaneously or preceding it by several days. The durations from onset to healing is less than 4 weeks ( -2 weeks). ${ }^{3,6,7}$

The initial diagnosis is entirely clinical, the important differential diagnosis being from a primary herpetic stomatitis may be confidently excluded since this is an isolated event in immuno-competent individuals. The involvement of the lips is a strong indication of the diagnosis of EM and the presence of "target" lesions of the skin can be taken as almost conclusive evidence for the diagnosis.

The histological appearances are variable. Widespread necrosis of keratinocytes with eosinophilic colloid change in the superficial epithelium may can be conspicuous. This may progress to intraepithelial vesicle or bulla formation. Degenerative changes in the epithelium are associated with infiltration by inflammatory cells which also involve the corium and may have a perivascular distribution. Leakage of immunoglobulin from blood vessels has been reported, but vasculitis not seen histologically. ${ }^{1}$ Patients should be warned of the possibility of recurrences but the disease usually runs a limited course.

As with adult, EM in the juvenile patient is managed with systemic corticosteroids. ${ }^{4}$ Treatment of the case is restricted to the mouth, depending on the use of local or systemic steroids. The use of systemic steroids for EM remains controversial due to the immune-suppressing side effect, it may give symptomatic relief. An initial dose of 30 to $50 \mathrm{mg}$ per day of prednisone or methyl-prednisolone for several days, which is then tapered, is helpful in shortening the healing time of EM, particularly when therapy is started early in the course of the disease. ${ }^{2,3,4,6}$ Antibiotics are often also prescribed in severe cases with the idea of preventing secondary infection.

There are two cases of EM described among children with varying forms of treatment and evolution. The diagnosis can be made based on characteristic clinical appearance accompanied by general complaints and medical history of the patient. Corticosteroids, topical and systemic, in spite of its immune-suppressing side effect, remains the drug of choice against this disease.

\section{REFERENCES}

1. Cawson RA, Odell EW. Essentials of oral pathology and oral medicine. $7^{\text {th }}$ ed. Toronto: Churchill Livingstone; 2005. p. 206-7.

2. Wray D, Lowe Gordon DO, Dagg JH, Felix DH, Scully C. Textbook of general and oral medicine. Edinburgh, London, New York, Philadelphia St Louis, Sydney, Toronto: Churchill Livingstone; 2001. p. 238-40.

3. Sen P, Chua SH. A case of recurrent erythema multiforme and its therapeutic complication: Case Report. J Ann Acad Med Singapore 2004; 33:793-6.

4. Pereira CM, Gaspaetto PF, Aires MP. Pempigus vulgaris in a juvenile patient: Case Report. J Oral Med Oral Diagnosis 2006 July-Agustus; 262-3.

5. Farthing PM, Maragaou P, Coates M, et al. Characteristics of the oral lesions in patients with cutaneous recurrent erythema multiforme. J Oral Pathol Med 1995; 24:9-13.

6. Greenberg M. Burket's oral medicine diagnosis and treatment. $10^{\text {th }}$ ed. Philadelphia: Lippincott-Reven; 2003. p. 57-59.

7. Gandolfo S, Scully C, Carrozo M. Oral medicine. Edinburgh London, NewYork, Philadelphia, St Louis, Sydney, Toronto: Churchill Livingstone; 2006. p. 72, 154. 